\title{
Alanine films for EPR dosimetry of low-energy (1-30 keV) X-ray photons
}

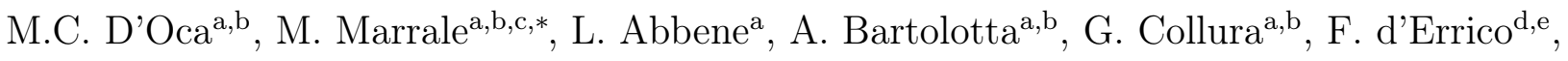 \\ F. Principato ${ }^{\mathrm{a}}$ \\ ${ }^{a}$ Department of Physics and Chemistry, University of Palermo, Viale delle Scienze, Edificio 18, I-90128 \\ Palermo, Italy \\ ${ }^{b}$ Istituto Nazionale di Fisica Nucleare (INFN), Via Santa Sofia, 64, I-95123 Catania, Italy \\ ${ }^{c}$ ATEN Center, University of Palermo Viale delle Scienze, Edificio 18, I-90128 Palermo, Italy \\ ${ }^{d}$ School of Engineering, University of Pisa, Largo Lazzarino 1, I-56126 Pisa, Italy \\ ${ }^{e}$ School of Medicine, Yale University, 333 Cedar St, New Haven CT 06520, United States
}

\begin{abstract}
L- $\alpha$-alanine has aroused considerable interest for use in radiation EPR dosimetry and has been formally accepted as a secondary standard for high-dose (kGy) and transfer dosimetry of high-energy photons and electrons. In this work, we extended the investigation of the energy response of alanine EPR films in the low energy range for X-photons (1-30 keV). Electron Paramagnetic Spin Resonance (EPR) measurements were performed on Kodak BioMax alanine films exposed to low-energy X-rays from a $\mathrm{Cu}-, \mathrm{W}$ - and Mo-targets tube operating at voltages up to $30 \mathrm{kV}$. Films were chosen because of the low penetration of the soft X-rays used. The response of alanine to low-energy X-rays was characterized experimentally and the relative response (with respect to high energy photons) was found to be between 0.8 and 0.9 for $\mathrm{Cu}$ - and $\mathrm{W}$ tube X-rays, and 1.0 for Mo-tube X-rays. The attenuation profiles were investigated and it was found that $1 \mathrm{~mm}$ of film material reduces the intensity of the X-ray-beam by about $70 \%, 50 \%$ and $40 \%$ for $\mathrm{Cu}-, \mathrm{W}$ - and Mo-tube X-rays, respectively. Monte Carlo simulations were performed to model the energy release as well as the depth dose profiles for the various radiation beams used. These data are considered relevant
\end{abstract}

\footnotetext{
${ }^{*}$ Corresponding author

Email address: maurizio.marrale@unipa.it (M. Marrale)
} 
for dosimetric applications in low energy beams such the high-gradient treatment fields used in monoenergetic microbeam radiation therapy (MRT) with synchrotron radiation as well as in brachytherapy with low energy sources, for instance ${ }^{169} \mathrm{Yb}$.

\section{Introduction}

Nowadays, electron paramagnetic resonance (EPR) dosimetry is widely employed for radiation measurements with several compounds such as ammonium tartrate [1 [5], lithium formate [6], dithionates, [7], phenolic compounds [8-12] and sugar [13, 14] and others [1519. However, crystalline L- $\alpha$-alanine is the most adopted material and is formally accepted as a secondary standard for high-dose measurements (kGy) and transfer dosimetry [2030]. Indeed, alanine has several favorable dosimetric features. First of all, the free radicals produced after irradiation are very stable and properly stored samples show negligible fading even one year after irradiation. Furthermore, the alanine response depends only slightly on various irradiation parameters such as photon energies above $100 \mathrm{keV}$, dose rate, and temperature. Moreover, the response is linear in a wide range of doses. Also, dosimeters can be prepared with small dimensions and have good mechanical resistance. The behavior is tissue equivalent for photon and electron beams with energies above $100 \mathrm{keV}$, whereas for lower energies the mass energy absorption coefficient of alanine is lower (up to 40\%) than that of soft tissue (for more details see Supplementary Figure 1). Finally, the readout procedure is non-destructive, contrary to most luminescent detectors [21, 26, 31-34]. In recent years, several studies have focused increasing the sensitivity of EPR dosimeters, reducing relaxation times and thus decreasing the microwave power saturation of the absorption line by adding high Z elements (such as silver, gold, rhodium, nickel, gadolinium) [3, 7, 23, 35 40].

Several reports describe the response of alanine to high energy photons and multiple dosimetric applications [21, 26, 31, 32, 41, 44]. Only scarce data are available on the response to medium energy X-rays. In particular, Zeng and McCaffrey worked at $150 \mathrm{kV}$ [45], Waldeland et al. investigated the response for tube voltages between $50 \mathrm{kV}$ and $200 \mathrm{kV}$ [46]. Anton and Büermann reported an extensive investigation on the relative response of alanine to x-rays with peak voltages between $30 \mathrm{kV}$ and $280 \mathrm{kV}$ [47. 
Our group previously reported the EPR response of alanine films exposed to low energy $\mathrm{X}$-rays produced with a $\mathrm{Cu}$-anode tube operating at $20 \mathrm{kV}$ [48]. Films were used due to the very low penetration of these soft X-rays causing strong gradients along the beam axis. In this work, we extended our analysis to soft X-rays produced with W-and Mo-targets and compared results to clinical LINAC X-rays. The absorbed dose was measured by means of an original spectroscopy system based on semiconductor detectors.

\section{Materials and methods}

\subsection{Sample preparation}

As in our previous work [48] we used commercial BioMax alanine films produced by Eastman Kodak (Rochester, NY, USA) and distributed by Bruker Biospin Italy, Milan. The sensitive layer of the dosimeter film is a mixture of polycrystalline $\mathrm{L}-\alpha$-alanine $(50 \%$ $\mathrm{w} / \mathrm{w}$ ) and a binder (a proprietary material of Eastman Kodak Company, USA) (50\% w/w) [48, 49]. According to the literature [49] the binder has molecular formula $\left(\mathrm{C}_{2} \mathrm{H}_{2} \mathrm{~F}_{2}\right)_{\mathrm{n}}\left(\mathrm{C}_{2} \mathrm{~F}_{4}\right)_{\mathrm{p}}$ assuming $\mathrm{n}=2$ and $\mathrm{p}=1$ and has a density of $1.88 \mathrm{~g} \mathrm{~cm}^{-3}$, whereas alanine has a density of $1.42 \mathrm{~g} \mathrm{~cm}^{-3}$. These films have a sensitive layer with a width of $4.0 \pm 0.1 \mathrm{~mm}$, a length of $45.0 \pm 0.2 \mathrm{~mm}$ and a thickness of $133.5 \pm 3.5 \mu \mathrm{m}$. The sensitive layer is attached to a Mylar backing with a thickness of $178 \mu \mathrm{m}$ [48, 49].

\subsection{Irradiations}

Irradiations of alanine films were performed at the DiFC "Livio Scarsi" Laboratory (LAX) of the University of Palermo, where a Seifert SN60 tube equipped with different targets $(\mathrm{Ag}, \mathrm{Co}, \mathrm{Cr}, \mathrm{Cu}, \mathrm{Fe}, \mathrm{Mo}$, and $\mathrm{W})$ is available to produce $\mathrm{X}$ rays in the $0.1-60$ $\mathrm{keV}$ energy range and with fluxes of $10^{5}-10^{8}$ photons $/ \mathrm{mm}^{2}$ [50]. In this work, X-rays were produced with $\mathrm{Cu}, \mathrm{W}$ and $\mathrm{Mo}$ targets and various tube voltage as reported in Table 1 . X-rays were produced to deliver dose up to 40 Gy to the BioMax alanine films using aluminium filter of $100 \mu \mathrm{m}$. More details on irradiations are reported in Table 1. For each dose value and anode type three films were irradiated. All irradiations were performed at room temperature. 
Table 1: Characteristis of low energy X-ray irradiations.

\begin{tabular}{cccccc}
\hline \hline Tube & $\begin{array}{c}\text { Current tube } \\
(\mathrm{mA}) \\
\text { Min-Max }\end{array}$ & Voltage tube $[\mathrm{kV}]$ & Filter & $\begin{array}{c}\text { Mean Energy } \\
(\mathrm{KeV})\end{array}$ & $\begin{array}{c}\text { Dose rate } \\
\text { Water })(\mathrm{Gy} / \mathrm{h}) \\
\text { Min-Max }\end{array}$ \\
\hline $\mathrm{Cu}$ & $2-80$ & 20 & $\mathrm{Al} 100 \mu \mathrm{m}$ & 10.5 & $0.107-4.26$ \\
$\mathrm{~W}$ & $2-68$ & 28 & $\mathrm{Al} 100 \mu \mathrm{m}$ & 13.9 & $0.295-10.00$ \\
$\mathrm{Mo}$ & $2-68$ & 28 & $\mathrm{Al} 100 \mu \mathrm{m}$ & 15.6 & $0.133-4.51$ \\
\hline \hline
\end{tabular}

The LAX facility is equipped with an original spectroscopy system based on semiconductor detectors (CdTe, Si) and digital pulse processing (DPP) electronics for X-ray spectra measurements. This device operates reliably even when exposed to high fluxes of photons [51, 52]. The $\mathrm{Cu}, \mathrm{W}$ and Mo-target X-ray spectra, measured with the this spectroscopy system are shown in Figure 1 .

The spectroscopy system was used to estimate the absorbed dose at the point of interest. In accordance with ASTM Standard E666-14 [53], the assessment was performed by folding the measured differential energy fluence distributions with tabulated mass energy absorption coefficients [54]. As it is widely recognized [55], this approach overcomes some critical issues: the energy dependence of a dosimeter response, the time-consuming calibration procedures and irradiation set-up constrains (X-ray tube target material, filters, field size, distance between the source and the measurement point, etc.), which derive from the particular irradiation set-up used in the calibration. Kerma values estimated with the digital detector system have a precision better than $2 \%$ (two standard deviation level). To assess the accuracy of the spectroscopy system, a commercial $\mathrm{NaI}(\mathrm{Tl})$ scintillator (AT1103M, ATOMTEX), working in the 5-160 keV energy range was used. At low current values (2 $\mathrm{mA}$ ) of the X-ray tube, air Kerma rates measured with the two systems are in agreement within the reported accuracy of the commercial dosimeter $( \pm 15 \%)$. Detection efficiency of the detectors and deadtime losses were taken into account in the dose estimation.

Alanine films were also irradiated with a $6 \mathrm{MV}$ photon beam produced by the linear accelerator employed for clinical treatments at Hospital "Villa Santa Teresa Diagnostica per Immagini e Radioterapia S.R.L." in Bagheria (near Palermo). Irradiations for doses up to 30 

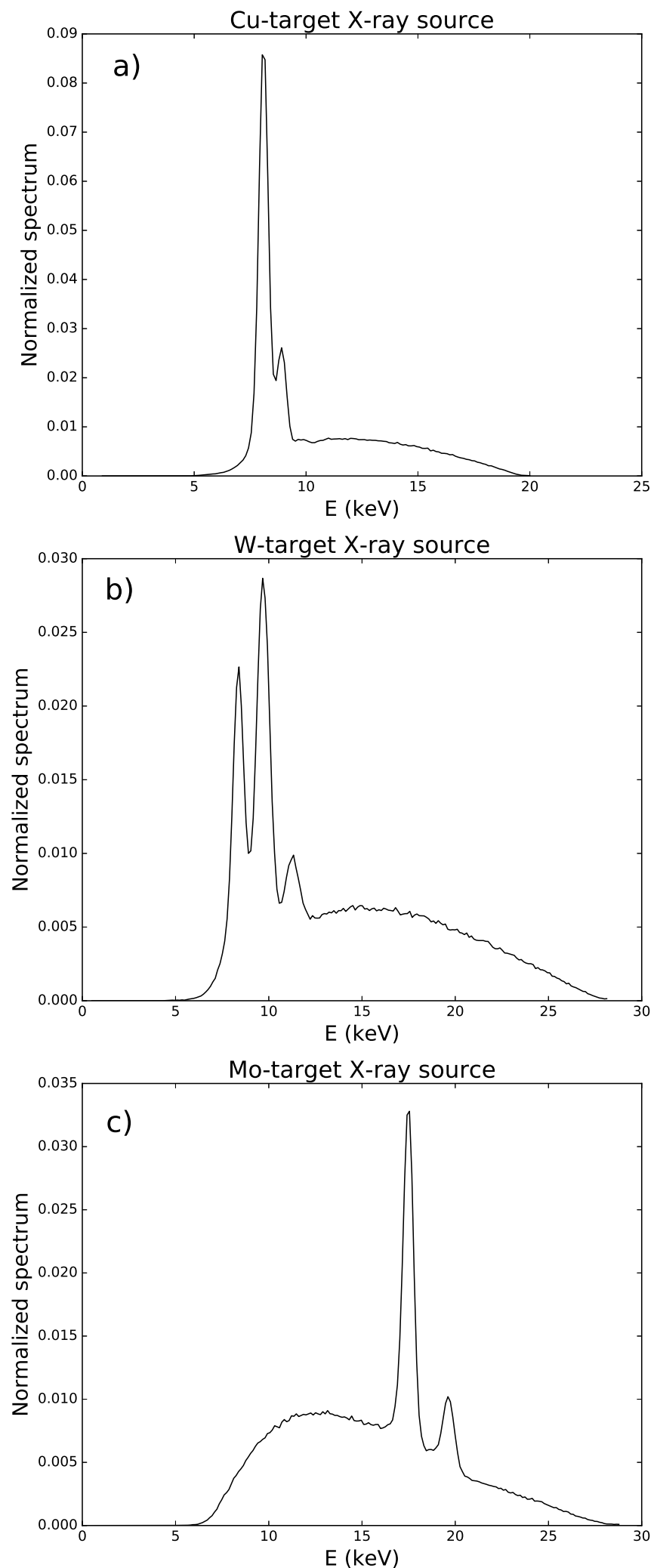

Figure 1: X-ray energy spectra of the beams used measured through the spectroscopy system described in the main text. 
Gy were carried out by placing films in a water equivalent phantom at the build-up depth. For each dose value, three films were irradiated.

\subsection{EPR measurements}

EPR measurements of the radiation-induced free radicals in alanine were done on a Bruker ELExysis E580 spectrometer equipped with a cylindrical High-Q cavity and operating in the X-band at approximately $9.8 \mathrm{GHz}$. The parameters chosen for these acquisitions were: microwave power of $2.99 \mathrm{~mW}$, a center field of $349.70 \mathrm{mT}$, a modulation amplitude of 1.00 $\mathrm{mT}$, a modulation frequency of $100 \mathrm{kHz}$, a sweep time of $40 \mathrm{~s}$ and 4 cumulated scans. The chosen field sweep was $3.0 \mathrm{mT}$ in order to detect only the central peak of the alanine spectra and avoid long acquisitions. The receiver gain were set to avoid signal distortions and yield the highest signal-to-noise ratio $(\mathrm{S} / \mathrm{N})$ [56, 57]. All measurements were performed at room temperature. In order to consider only the EPR signal due to irradiation the average signal of unirradiated samples was subtracted from the spectra of irradiated samples. The peak-topeak amplitude $\left(h_{p p}\right)$ of the central line of the difference spectrum was chosen as dosimetric parameter. The film strips were inserted in Suprasil ${ }^{\circledR}$ quartz tubes with a $5 \mathrm{~mm}$ inner diameter and placed at the center of the microwave cavity in order to maximize the EPR signal (see Figure 2).

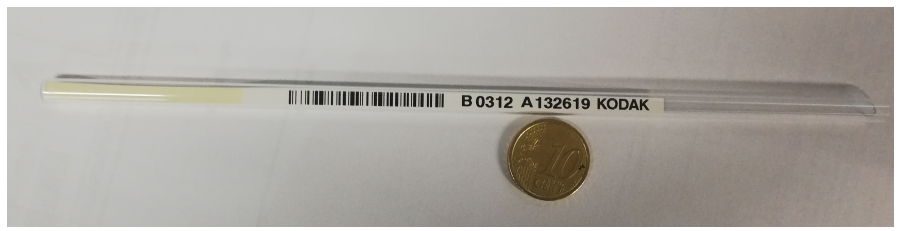

Figure 2: Picture of the film inserted inside the quartz for the EPR measurements.

In order to average out the EPR signal amplitude dependence on the orientation of the films inside the resonant cavity, each sample was read out four times rotating it by $90^{\circ}$. Four orientations at $90^{\circ}$ are easy to implement and previous measurements at more orientations produced a comparable standard deviation. Three dosimeters were irradiated to each dose value, and the signal was divided by the mass of alanine. The resulting 12 values of $h_{r}$ (four orientations $\times$ three dosimeters) were averaged in order to reduce uncertainties, i.e. 


\begin{tabular}{cccc}
\hline \hline Element & Alanine & Binder & Mylar \\
\hline $\mathrm{H}$ & 0.07865 & 0.01818 & 0.0417 \\
$\mathrm{C}$ & 0.40449 & 0.32727 & 0.6250 \\
$\mathrm{~N}$ & 0.15730 & - & - \\
$\mathrm{O}$ & 0.35955 & - & 0.3333 \\
$\mathrm{~F}$ & - & 0.65455 & - \\
\hline \hline
\end{tabular}

Table 2: Atomic composition of the materials used $(\% \mathrm{w} / \mathrm{w})$. These values were considered in the MC simulations.

$h_{r}=\frac{1}{12} \sum_{j=1}^{3} \sum_{i=1}^{4} \frac{h_{p p_{j i}}}{m_{j}}$. Calibration curves were then built as $h_{r}$ versus absorbed dose in water.

\subsection{Monte Carlo simulations}

In order to model the experimental EPR results Monte Carlo simulations were performed. In particular, the average energy deposited inside alanine was estimated by means of the Monte Carlo N-particle radiation transport code MCNP5 [58]. For these simulations the films were modelled as two different layers: one for alanine plus binder and the other for the mylar support. The layer of alanine with binder was assumed to be a homogeneous mixture of $50 \%$ alanine and $50 \%$ binder (with average density of $1.62 \mathrm{~g} / \mathrm{cm}^{3}$ ) with a thicknees of $133.5 \mu \mathrm{m}$. The mylar layer was assumed to have a density of $1.455 \mathrm{~g} / \mathrm{cm}^{3}$ to be thick 178 $\mu \mathrm{m}$. The atomic compositions of the various materials considered in the $\mathrm{MC}$ simulations are reported in Table 2 .

Two different geometry setups were considered for the two different irradiation modalities: low energy X-ray beam and LINAC beam. In the case of X-tube ray beam the various alanine films were considered as stacked in order to investigate the depth dose profile (as also investigated experimentally). The spectra of the X-ray beam are those shown in Figure 1. In the case of LINAC irradiation the alanine film was placed between two water layers at the depth of build-up position. In this case the spectrum used was that produced by a LINAC at $6 \mathrm{MV}$ and it is composed mainly of photons with energy of the order of $1 \mathrm{MeV}$ (for more details see [23]). The tally considered is the energy released inside the sensitive material layer. Furthermore, in order to investigate the effect of the binder on the film sensitivity 
other MC simulations were performed considering the sensitive layer as composed of pure alanine (instead of alanine and binder) and with a density equal to the effective density of sensitive layer of the film (i.e. average density $=1.62 \mathrm{~g} / \mathrm{cm}^{3}$ ). Ten million histories were considered for each Monte Carlo run.

\section{Results and discussion}

The central part of the EPR spectra of alanine films exposed to 50 Gy of low-energy Xrays are similar to those for high energy photons (see Supplementary Figure 2). Since alanine dosimeters exposed to photon beams with different energies show different relative amounts of the free radicals induced by radiations [59], an investigation of the spectra was performed to evaluate possible differences with beam energies. For this analysis entire spectra (field sweep $20 \mathrm{mT}$ ) of film alanine samples exposed to various energies (at $30 \mathrm{~Gy}$ ) were acquired with a modulation amplitude of $0.2 \mathrm{mT}$ in order to achieve a compromise between spectral resolution and signal intensity. The resulting spectra are reported in Figure 3 . For the dose values considered and the acquisition parameters used no significative differences were observed among the various samples.

Figure 4 shows the EPR signals of alanine film samples irradiated with $\mathrm{Cu}-, \mathrm{W}$ - and Mo-target X-rays to dose of 30 Gy taking as reference the signal of samples exposed to 6 MV LINAC photons.

As shown in Figure 4, the EPR signals for low energy photons are lower than or equal to the signal for clinical LINAC beams (within the experimental uncertainties). In particular, for $\mathrm{Cu}$ - and $\mathrm{W}$ - targets the relative EPR response takes values between 0.8 and 0.9 (a similar trend was observed for films exposed at $20 \mathrm{~Gy}$, see Supplementary Figure 3). Therefore, the investigated films show an energy dependent response for these beams.

As possible causes of the reduction of the EPR signal with decreasing beam energies we considered two independent factors: i) the effect of the binder on the samples and ii) the decrease of number of radicals per unit absorbed dose at low photon energies, as reported in literature [45, 47, 60]. In order to evaluate the effect of the binder, Monte Carlo simulations were perfomed following an analogous procedure as reported in the work of [60] 


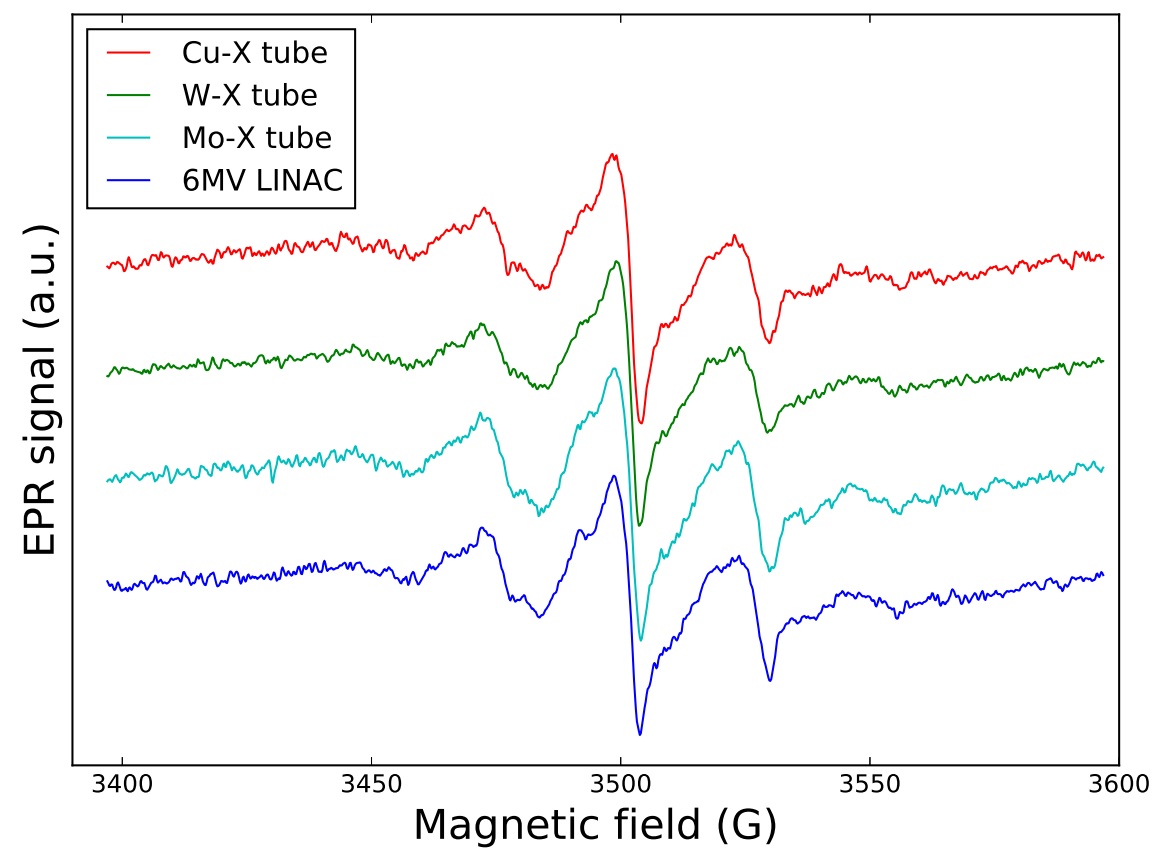

Figure 3: Comparison of entire EPR spectra of alanine film samples irradiated at 30 Gy with $\mathrm{Cu}-$, W- and Mo-target X-rays and $6 \mathrm{MV}$ LINAC photons (modulation amplitude: $0.2 \mathrm{mT}$ ).

and, therefore, considering two cases: a) the sensitive layer is composed of alanine and binder (as it effectively is) and b) the sensitivity layer is composed of only alanine. In particular, in the case a) the effective density of $1.62 \mathrm{~g} / \mathrm{cm}^{3}$ and the sensitive layer is composed of equal masses of alanine and binder. In the case b) alanine is considered as sensitive material and binder but with a reduced density so that the true density of the sensitive layer (1.62 $\mathrm{g} / \mathrm{cm}^{3}$ ) was hold. Indeed, according to the Fano theorem, this last case is equivalent to assuming that the alanine grains (with density of $1.42 \mathrm{~g} / \mathrm{cm}^{3}$ ) were suspended in a matrix of alanine of the same density $\left(1.88 \mathrm{~g} / \mathrm{cm}^{3}\right)$ as that of the binder. The energy released in the sensitive layer was obtained through $\mathrm{MC}$ for both configurations and the ratio between the energy released in the case of alanine plus binder and the energy released in the case of pure alanine was estimated. Since the mass of the sensitive layer is the same in both cases, this ratio is equal to the ratio of absorbed doses to the sensitive layer. In order to obtain the mean absorbed dose in the sensitive alanine material of the film $\overline{\mathrm{D}}_{\text {det }}$ we used two different expressions for the two different photon energy ranges considered. In particular, for low 


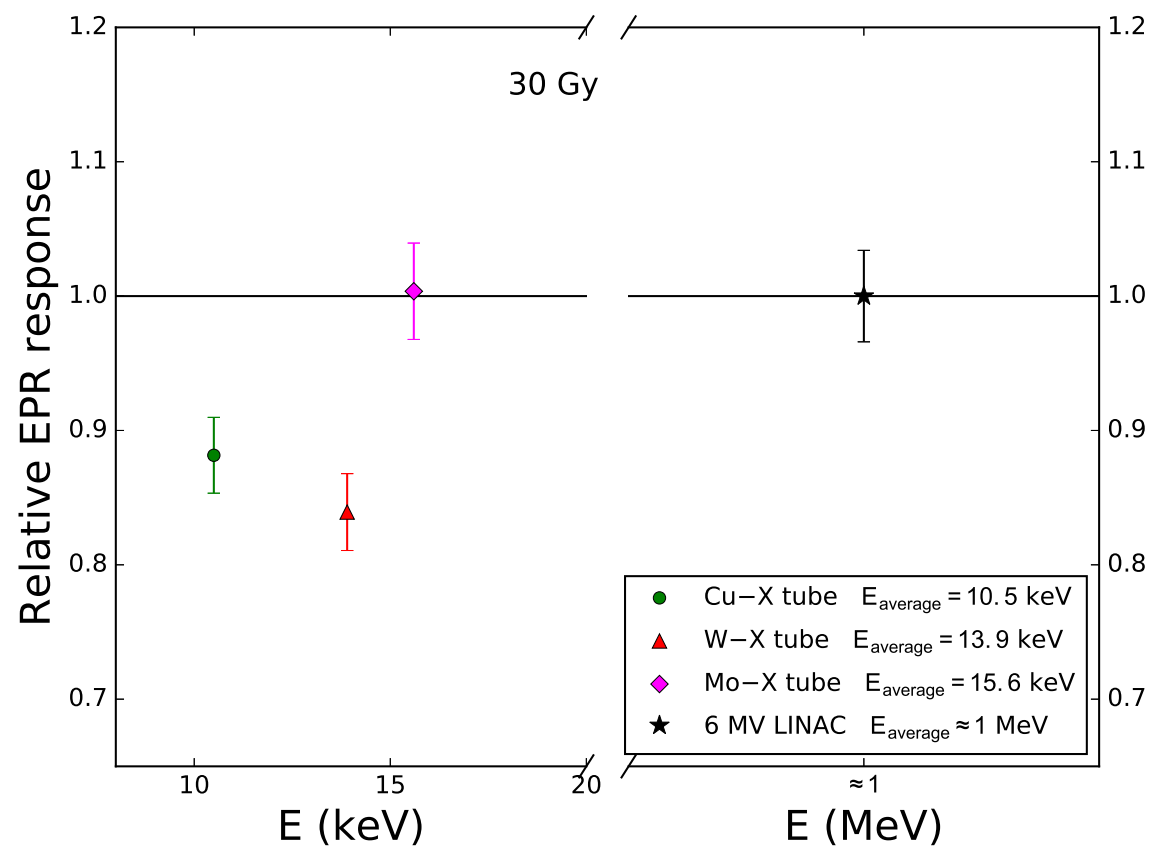

Figure 4: EPR signal of alanine film samples irradiated to $30 \mathrm{~Gy}$ with $\mathrm{Cu}-$, W- and Mo-target X-rays normalized to the signal of samples exposed to 6 MV LINAC photons.

energy photons, under the hypothesis that most of secondary electrons produced in alanine grains release energy inside the same grains, the mean absorbed dose in the homogeneous mixture of alanine and binder obtained through the MC simulations, $\left[\overline{\mathrm{D}}_{\text {det }}\right]_{\mathrm{MC} \text {,mixture, was }}$ corrected using the following expression (adapted from [60]):

$$
\left[\overline{\mathrm{D}}_{\mathrm{det}}\right]=\left[\overline{\mathrm{D}}_{\text {det }}\right]_{\mathrm{MC}, \text { mixture }} \frac{\mathrm{m}_{\mathrm{det}}}{\mathrm{m}_{\text {alanine }}+\left[\overline{\frac{\mu_{\mathrm{en}}}{\rho}}\right]_{\text {alanine }}^{\text {binder }} \mathrm{m}_{\text {binder }}}
$$

where $\mathrm{m}_{\mathrm{det}}$ is the total mass of the sensitive layer, $\mathrm{m}_{\text {alanine }}=\mathrm{m}_{\text {binder }}=\mathrm{m}_{\mathrm{det}} / 2, \overline{\overline{\mathrm{en}_{\mathrm{n}}}}$ is the average value of the ratio of the mass-energy absorption coefficients of the two materials. These last values were retrieved from the NIST database and a plot of this ratio as function of energy is reported in Supplementary Figure 4. According to this plot an average value of this ratio in the energy range of interest for the low energy X-ray beams (between 5 and $20 \mathrm{keV}$ ) is 1.7. In the case of high energy photons produced by LINAC, since secondary electrons produced inside the detector pass through alanine grains and binder grains, the 


\begin{tabular}{lcc}
\hline \hline Beam & $E_{\text {ala }+ \text { binder }} / E_{\text {ala }}$ & $\overline{\mathrm{D}}_{\text {det }} / \overline{\mathrm{D}}_{\text {det,pure alanine }}$ \\
\hline Cu-target X-rays & $1.35 \pm 2 \%$ & $1.00 \pm 2 \%^{1}$ \\
W-target X-rays & $1.36 \pm 2 \%$ & $1.01 \pm 2 \%^{1}$ \\
Mo-target X-rays & $1.37 \pm 2 \%$ & $1.02 \pm 2 \%^{1}$ \\
6MV LINAC rays & $0.98 \pm 2 \%$ & $0.99 \pm 2 \%^{2}$ \\
\hline
\end{tabular}

Table 3: Ratio between the energy released in the case of alanine plus binder and the energy released in the case of pure alanine for all photon beams used. ${ }^{1}$ Value calculated using Eq. 1. ${ }^{2}$ Value calculated using Eq. 2 .

correction of the mean absorbed dose in the homogeneous mixture of alanine and binder

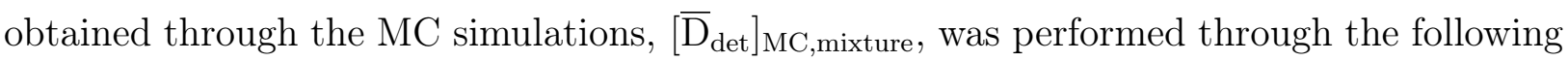
expression taking into account the mass electron stopping power (adapted from [60]):

$$
\left[\overline{\mathrm{D}}_{\text {det }}\right]=\left[\overline{\mathrm{D}}_{\text {det }}\right]_{\text {MC,mixture }} \frac{\mathrm{m}_{\text {det }}}{\mathrm{m}_{\text {alanine }}+\frac{\bar{\Phi}_{\text {binder }}}{\bar{\Phi}_{\text {alanine }}}\left[m \bar{s}_{\text {col }}\right]_{\text {alanine }}^{\text {binder }} \mathrm{m}_{\text {binder }}}
$$

where $\mathrm{m}_{\mathrm{det}}$ is the total mass of the sensitive layer, $\mathrm{m}_{\text {alanine }}=\mathrm{m}_{\text {binder }}=\mathrm{m}_{\mathrm{det}} / 2$, $\bar{\Phi}_{\text {binder }}$ and $\bar{\Phi}_{\text {alanine }}$ are the mean electron fluences in the alanine grains and in the binder matrix, respectively. The value of $\bar{\Phi}_{\text {binder }}$ is larger to $\bar{\Phi}_{\text {alanine }}$ because of the higher scattering power of medium with higher effective atomic number Z, and, analogously to [60], $\bar{\Phi}_{\text {binder was }}$ assumed to be $1.10 \bar{\Phi}_{\text {alanine }}\left[{ }_{m} \bar{s}_{\text {col }}\right]_{\text {alanine }}^{\text {binder }}$ is the average value of the ratio of the mass electron stopping power coefficients of the two materials. A plot of this ratio as function of energy is reported in Supplementary Figure 5. According to this plot an average value of this ratio in the energy range of interest for the LINAC X-ray photons (between 0.1 and $6 \mathrm{MeV}$ ) is 0.885 .

The results of this analysis are reported in Table 3. In the last column the ratio $\overline{\mathrm{D}}_{\text {det }} / \overline{\mathrm{D}}_{\text {det,pure alanine }}$ is reported. From both Eqs. 1 and 2 it is evident that $\overline{\mathrm{D}}_{\text {det,pure alanine }}=$ $\overline{\mathrm{D}}_{\mathrm{MC}, \text { pure alanine. }}$

As can be seen from Table 3 , the presence of binder causes a negligible effect on the energy released in the sensitive layer for high energy photons, whereas it induces an increment of the energy released inside this layer for low energy photons. This can be explained by considering that for these alanine films the binder has an effective atomic number (calculated 
as the weighted average of the atomic numbers, i.e. $\left.Z_{\text {eff }}=\left(\sum_{i} m_{i} Z_{i}\right) /\left(\sum_{i} m_{i}\right)\right)$ is 6.48 for alanine and 7.87 for the binder and the mass energy absorption coefficient of the binder is $\sim 1.7$ times larger than that of alanine for low energy photons. This involves that for low energy beam an increase of energy release in the sensitive layer could be expected. However, if the mean absorbed dose in the alanine grains in the binder matrix is compared to the mean absorbed dose in the alanine grains suspended in an alanine matrix only variations up to $2 \%$ are observed in this energy range and of about $1 \%$ for high energy photons. Therefore, the presence of the binder involves a very small increment (however within uncertainty) of dose in alanine due to the larger mass energy absorption coefficient of the binder for low energy photons. A change in sensitivity due to the binder was also observed previously in literature [60]. In that work the Authors found an increase of energy imparted to the dosimeters when suspended in paraffin wax compared to if suspended in lithium formate for photon energies below $30 \mathrm{keV}$ with a maximum of $6 \%$ at $10 \mathrm{keV}$ explained by the higher mass collision stopping power for paraffin compared to lithium formate. In the present case the effective atomic number of the binder larger than that of alanine as well as the high concentration of the binder $(50 \% \mathrm{w} / \mathrm{w})$ increase the dose absorbed by the sensitive layer even though does not increase significantly the dose in alanine (and therefore the EPR signal). For high energy photons the effect of the binder is negligible because for energies of the order of $1 \mathrm{MeV}$ the mass energy absorption coefficients of alanine and binder differ few percents each other. Consequently, the presence of the binder does not justify the sensitivity decrease observed for low photon energies. Therefore, the sensitivity reduction for lower photon energies could be attributed to a decrease of the number of radicals per unit absorbed dose. Hence, we conclude that the main effect is the dependence of the LET on the energy and the response of alanine samples to these low energy photons is lower than to high energy photons. A comparison of these results with literature data for alanine pellets exposed to slightly higher energies (the lowest energy value considered was $19.3 \mathrm{keV}$ ) [45 47, 60] highlights that the reduction of the relative response is higher for pellets (for which it can drop to about 0.65) than for films. One possible reason for this could be that the pellets used by Anton et al. have a diameter of $4.8 \mathrm{~mm}$ and an height of $2.8 \mathrm{~mm}$ and this extended volume is more prone 
to remain partially unirradiated by low-energy photons with respect to the films which have a thickness of about $130 \mu \mathrm{m}$. Furthermore, Figure 4 shows that the relative response of samples exposed to Mo-anode tube is not significantly different (within 1 S.D.) from that to high energy clinical photons.

\section{Beam attenuation}

The attenuation of the X-ray beam through the dosimeter was examined using stacked alanine films. These comprise a $178 \mu \mathrm{m}$ thick mylar layer and a $135.5 \mu \mathrm{m}$ thick alanine layer. Ten stacked BioMax films were placed perpendicular to the radiation beam and exposed to an entrance dose of 40 Gy for the various low energy radiation beams used. These doses are comparable to those used in the high-gradient treatment fields used in monoenergetic microbeam radiation therapy (MRT) with synchrotron radiation [61] as well as in brachytherapy with low energy sources, for instance ${ }^{169} \mathrm{Yb}[62$.

Figure 5 reports the results of this analysis. The signal presents a rapid drop by about $70 \%, 50 \%$ and $40 \%$ within $1 \mathrm{~mm}$ for $\mathrm{Cu}-, \mathrm{W}$ - and Mo-tube X-rays, respectively. As expected a higher penetration in alanine is observed with increasing beam energy. At a depth greater than $1 \mathrm{~mm}$, a much slower decrease and leveling out of the curves is observed, reaching a value of about $0.2,0.3$ and 0.5 for for $\mathrm{Cu}_{-}, \mathrm{W}$ - and Mo-tube $\mathrm{X}$-rays, respectively. As stated above, the attenuation of these low-energy X-rays (mostly $\mathrm{Cu}$-anode $\mathrm{X}$-rays) quickly reduces the dose levels below minimum detectable values; therefore, thin films must be used for these low energy beams, rather than thicker pellets.

In order to model the depth dose profile with these films for low energy photons we have carried out MC simulations considering the alanine films stacked along the propagation direction of the low energy X-ray beams for all targets used. The results are shown as a continuous line in Figure 5. As can be seen from this Figure there is a very good agreement between Monte Carlo simulations and experimental values. 


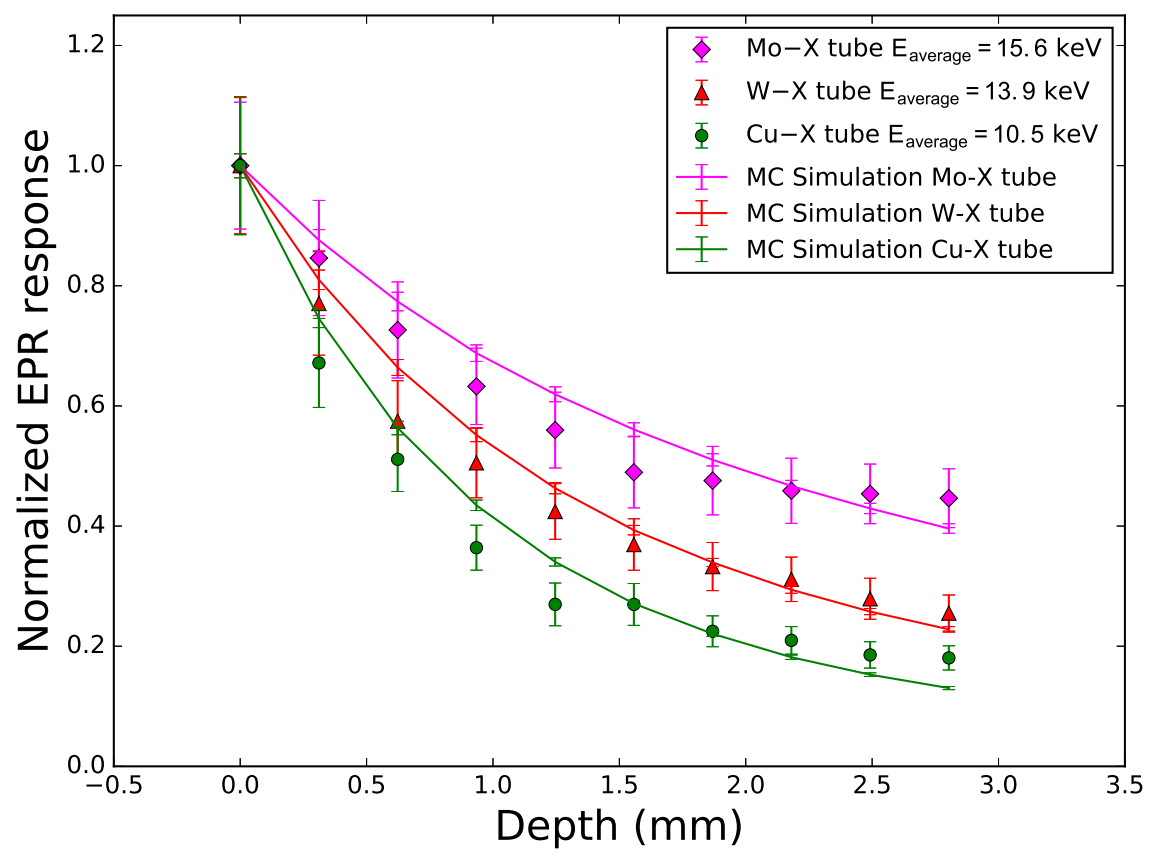

Figure 5: Normalized EPR response of commercial alanine films as function of depth for the low-energy photon beams used.

\section{Conclusions}

Commercial Kodak BioMax alanine films were exposed to $\mathrm{Cu}-, \mathrm{W}$ - and Mo-target Xtube photons for doses up to $40 \mathrm{~Gy}$ and the relative response (with respect to high energy photons) was found to be between 0.8 and 0.9 for $\mathrm{Cu}$ - and $\mathrm{W}$-tube X-rays, and 1.0 Mo-tube X-rays. The attenuation profiles were investigated since these beams are rapidly attenuated: $1 \mathrm{~mm}$ of film material reduces their intensity by about $70 \%, 50 \%$ and $40 \%$ within $1 \mathrm{~mm}$ for $\mathrm{Cu}-, \mathrm{W}$ - and Mo-tube X-rays, respectively. These results were modelled through Monte Carlo simulations. Therefore, the $\sim 100$ micron active thickness of the Kodak BioMax alanine films is very useful in the measurement of these low energy X-Rays and they are promising for the dosimetry of low energy synchrotron radiation in the form of microplanar beams for the treatment of brain metastases as well as in brachytherapy with low energy sources. 


\section{Declaration of Competing Interest}

The authors declare that they have no known competing financial interests or personal relationships that could have appeared to influence the work reported in this paper.

\section{Acknowledgments}

This research was supported in part by grants PRIN 2010SNALEM and PRIN 2012WM9MEP of the Italian Ministry for Education, University and Research (MIUR). The Authors thank Calogero Rinaldi for irradiation of alanine films with a $6 \mathrm{MV}$ photon beam produced by the linear accelerator employed for clinical treatments at Hospital "Villa Santa Teresa Diagnostica per Immagini e Radioterapia S.R.L." in Bagheria.

\section{Appendix A. Supplementary data}

Supplementary data associated with this article can be found, in the online version, at https://doi.org/10.1016/j.nimb.2019.08.011.

\section{References}

[1] M. Marrale, M. Brai, A. Barbon, M. Brustolon, Analysis of the spatial distribution of free radicals in ammonium tartrate by pulse EPR techniques, Radiation Research 171 (3) (2009) 349-359.

[2] M. Marrale, S. Basile, M. Brai, A. Longo, Monte Carlo simulation of the response of ESR dosimeters added with gadolinium exposed to thermal, epithermal and fast neutrons, Applied Radiation and Isotopes 67 (7-8 SUPPL.) (2009) 186-189.

[3] M. Marrale, M. Brai, A. Longo, S. Panzeca, L. Tranchina, E. Tomarchio, A. Parlato, A. Buttafava, D. Dondi, Neutron ESR dosimetry through ammonium tartrate with low Gd content, Radiation protection dosimetry 159 (1-4) (2014) 233-236.

[4] M. Brustolon, F. Tampieri, M. Marrale, A. Barbon, Determination of new radical species in ammonium tartrate dosimeters by cw-and pulsed-EPR techniques, Applied Magnetic Resonance 46 (4) (2015) 481488.

[5] M. Marrale, A. Longo, M. Brai, A. Barbon, M. Brustolon, Discrimination of Radiation Quality Through Second Harmonic Out-of-Phase cw-ESR Detection, Radiation research 181 (2) (2014) 184-192. 
[6] E. Lund, H. Gustafsson, M. Danilczuk, M. Sastry, A. Lund, T. Vestad, E. Malinen, E. Hole, E. Sagstuen, Formates and dithionates: sensitive EPR-dosimeter materials for radiation therapy, Applied radiation and isotopes 62 (2) (2005) 317-324.

[7] H. Gustafsson, M. Danilczuk, M. Sastry, A. Lund, E. Lund, Enhanced sensitivity of lithium dithionates doped with rhodium and nickel for EPR dosimetry, Spectrochimica Acta Part A: Molecular and Biomolecular Spectroscopy 62 (1) (2005) 614-620.

[8] M. Marrale, A. Longo, S. Panzeca, S. Gallo, F. Principato, E. Tomarchio, A. Parlato, A. Buttafava, D. Dondi, A. Zeffiro, ESR response of phenol compounds for dosimetry of gamma photon beams, Nuclear Instruments and Methods in Physics Research Section B: Beam Interactions with Materials and Atoms 339 (2014) 15-19.

[9] M. Marrale, S. Gallo, A. Longo, S. Panzeca, A. Parlato, A. Buttafava, D. Dondi, A. Zeffiro, Study of the response of phenol compounds exposed to thermal neutrons beams for Electron Paramagnetic Resonance dosimetry, Radiation Measurements 75 (2015) 15-20.

[10] S. Gallo, S. Panzeca, A. Longo, S. Altieri, A. Bentivoglio, D. Dondi, R. Marconi, N. Protti, A. Zeffiro, M. Marrale, Testing and linearity calibration of films of phenol compounds exposed to thermal neutron field for EPR dosimetry, Applied Radiation and Isotopes 106 (2015) 129-133.

[11] S. Gallo, G. Iacoviello, A. Bartolotta, D. Dondi, S. Panzeca, M. Marrale, ESR dosimeter material properties of phenols compound exposed to radiotherapeutic electron beams, Nuclear Instruments and Methods in Physics Research Section B: Beam Interactions with Materials and Atoms 407 (2017) 110117.

[12] S. Gallo, G. Iacoviello, S. Panzeca, I. Veronese, A. Bartolotta, D. Dondi, A. M. Gueli, G. Loi, A. Longo, E. Mones, et al., Characterization of phenolic pellets for ESR dosimetry in photon beam radiotherapy, Radiation and environmental biophysics 56 (4) (2017) 471-480.

[13] A. Gutierrez, J. Azorin, Lyoluminescence and electron spin resonance of commercial sugar in accident dosimetry, Nucl. Sci. J 30 (1993) 137.

[14] F. d'Errico, P. Fattibene, S. Onori, M. Pantaloni, Criticality accident dosimetry with ESR spectroscopy, Applied Radiation and Isotopes 47 (11) (1996) 1335-1339.

[15] M. O. Bal, H. Tuner, ESR dosimetry and radical kinetics of gamma-irradiated propyl gallate, Journal of Molecular Structure 1071 (2014) 123-127.

[16] H. Tuner, M. A. Kayıkçı, EPR investigation of thermal decay of radiation-induced species of benzoic acid and its sodium and potassium salts, Radiation and environmental biophysics 54 (2) (2015) 243-249.

[17] A. Longo, S. Basile, M. Brai, M. Marrale, L. Tranchina, ESR response of watch glasses to proton beams, Nuclear Instruments and Methods in Physics Research Section B: Beam Interactions with Materials and Atoms 268 (17-18) (2010) 2712-2718. 
[18] M. Marrale, A. Longo, M. Brai, A. Barbon, M. Brustolon, P. Fattibene, Pulsed EPR analysis of tooth enamel samples exposed to UV and $\gamma$-radiations, Radiation Measurements 46 (9) (2011) 789-792.

[19] M. Marrale, A. Longo, M. D'oca, A. Bartolotta, M. Brai, Watch glasses exposed to 6 MV photons and $10 \mathrm{MeV}$ electrons analysed by means of ESR technique: A preliminary study, Radiation Measurements 46 (9) (2011) 822-826.

[20] M. Ikeya, New applications of electron spin resonance: dating, dosimetry and microscopy, World Scientific, 1993

[21] D. F. Regulla, ESR spectrometry: a future-oriented tool for dosimetry and dating, Appl. Radiat. lsot. 62 (2005) 117-127.

[22] M. Brai, G. Gennaro, M. Marrale, A. Bartolotta, M. D’Oca, ESR response to $\gamma$-rays of alanine pellets containing $\mathrm{B}(\mathrm{OH})_{3}$ or $\mathrm{Gd}_{2} \mathrm{O}_{3}$, Applied Radiation and Isotopes 65 (4) (2007) 435-439.

[23] M. Marrale, A. Longo, M. Spanò, A. Bartolotta, M. D’Oca, M. Brai, Sensitivity of alanine dosimeters with gadolinium exposed to $6 \mathrm{MV}$ photons at clinical doses, Radiation research 176 (6) (2011) 821-826.

[24] F. Trompier, C. Huet, R. Medioni, I. Robbes, B. Asselineau, Dosimetry of the mixed field irradiation facility CALIBAN, Radiation Measurements 43 (2) (2008) 1077-1080.

[25] E. Malinen, EPR Dosimetry in Clinical Applications, in: Applications of EPR in Radiation Research, Springer, 2014, pp. 509-538.

[26] O. Baffa, A. Kinoshita, Clinical applications of alanine/electron spin resonance dosimetry, Radiation and environmental biophysics (2014) 1-8.

[27] R. B. Hayes, E. H. Haskell, A. Wieser, A. A. Romanyukha, B. L. Hardy, J. K. Barrus, Assessment of an alanine EPR dosimetry technique with enhanced precision and accuracy, Nuclear Instruments and Methods in Physics Research Section A: Accelerators, Spectrometers, Detectors and Associated Equipment 440 (2) (2000) 453-461.

[28] E. A. Ainsbury, D. Samaga, S. Della Monaca, M. Marrale, C. Bassinet, C. I. Burbidge, V. Correcher, M. Discher, J. Eakins, P. Fattibene, et al., Uncertainty on radiation doses estimated by biological and retrospective physical methods, Radiation protection dosimetry 178 (4) (2017) 382-404.

[29] H. Tuner, M. Korkmaz, Kinetic features of the radical species produced in $\gamma$-irradiated dl-tartaric acid and the dosimetric potential of this acid, Radiation research 172 (1) (2009) 120-128.

[30] A. Carlino, C. Gouldstone, G. Kragl, E. Traneus, M. Marrale, S. Vatnitsky, M. Stock, H. Palmans, End-to-end tests using alanine dosimetry in scanned proton beams, Physics in Medicine \& Biology 63 (5) (2018) 055001.

[31] F. Coninckx, H. Schönbacher, A. Bartolotta, S. Onori, A. Rosati, Alanine dosimetry as the reference dosimetric system in accelerator radiation environments, International Journal of Radiation Applications and Instrumentation. Part A. Applied Radiation and Isotopes 40 (10) (1989) 977-983. 
[32] D. F. Regulla, From dating to biophysics - 20 years of progress in applied esr spectroscopy, Appl. Radiat. lsot. 52 (2000) 1023-1030.

[33] C. De Angelis, A. Soriani, M. Benassi, S. Onori, On measuring the output of an IORT mobile dedicated accelerator, Radiation protection dosimetry 120 (1-4) (2006) 221-225.

[34] M. Marrale, A. Longo, G. Russo, C. Casarino, G. Candiano, S. Gallo, A. Carlino, M. Brai, Dosimetry for electron Intra-Operative RadioTherapy: Comparison of output factors obtained through alanine/EPR pellets, ionization chamber and Monte Carlo-GEANT4 simulations for IORT mobile dedicate accelerator, Nuclear Instruments and Methods in Physics Research Section B: Beam Interactions with Materials and Atoms 358 (2015) 52-58.

[35] G. M. Hassan, M. Ikeya, Metal ion-organic compound for high sensitive ESR dosimetry, Applied Radiation and Isotopes 52 (5) (2000) 1247-1254.

[36] M. Danilczuk, H. Gustafsson, M. Sastry, E. Lund, Development of nickel-doped lithium formate as potential EPR dosimeter for low dose determination, Spectrochimica Acta Part A: Molecular and Biomolecular Spectroscopy 67 (5) (2007) 1370-1373.

[37] F. Chen, P. Nicolucci, O. Baffa, Enhanced sensitivity of alanine dosimeters to low-energy X-rays: Preliminary results, Radiation Measurements 43 (2) (2008) 467-470.

[38] F. Chen, J. V. Ramirez, P. Nicolucci, O. Baffa, Sensitivity comparison of two L-alanine doped blends to different photon energies, Health physics 98 (2) (2010) 383-387.

[39] E. J. Guidelli, A. P. Ramos, M. E. D. Zaniquelli, P. Nicolucci, O. Baffa, Synthesis of silver nanoparticles using DL-alanine for ESR dosimetry applications, Radiation Physics and Chemistry 81 (3) (2012) 301307.

[40] M. Marrale, T. Schmitz, S. Gallo, G. Hampel, A. Longo, S. Panzeca, L. Tranchina, Comparison of EPR response of alanine and $\mathrm{Gd}_{2} \mathrm{O}_{3}$-alanine dosimeters exposed to TRIGA Mainz reactor, Applied Radiation and Isotopes 106 (2015) 116-120.

[41] K. Schultka, B. Ciesielski, K. Serkies, T. Sawicki, Z. Tarnawska, J. Jassem, EPR/alanine dosimetry in LDR brachytherapyâĂŤa feasibility study, Radiation protection dosimetry 120 (1-4) (2006) 171-175.

[42] B. Ciesielski, M. Tyszkowska, A. Grudniewska, M. Penkowski, K. Schultka, Z. Peimel-Stuglik, The effect of dose on light-sensitivity of radicals in alanine EPR dosimeters, Spectrochimica Acta Part A: Molecular and Biomolecular Spectroscopy 69 (5) (2008) 1405-1416.

[43] K. Schultka, B. Ciesielski, K. Serkies, B. Wysocka, T. Sawicki, Z. Tarnawska, J. Jassem, In vivo dosimetry using electron paramagnetic resonance in L-alanine in gynecological low dose rate brachytherapy, Nowotwory. Journal of Oncology 54 (6) (2004) 560-560.

[44] E. S. Bergstrand, K. R. Shortt, C. K. Ross, E. O. Hole, An investigation of the photon energy dependence of the EPR alanine dosimetry system, Physics in Medicine \& Biology 48 (12) (2003) 1753. 
[45] G. Zeng, J. McCaffrey, The response of alanine to a $150 \mathrm{keV}$ X-ray beam, Radiation physics and chemistry 72 (5) (2005) 537-540.

[46] E. Waldeland, E. O. Hole, E. Sagstuen, E. Malinen, The energy dependence of lithium formate and alanine EPR dosimeters for medium energy x rays, Medical physics 37 (7) (2010) 3569-3575.

[47] M. Anton, L. Büermann, Relative response of the alanine dosimeter to medium energy X-rays, Physics in medicine and biology 60 (15) (2015) 6113.

[48] M. Marrale, L. Abbene, F. d'Errico, S. Gallo, A. Longo, S. Panzeca, L. Tana, L. Tranchina, F. Principato, Characterization of the ESR response of alanine dosimeters to low-energy Cu-target X-tube photons, Radiation Measurements 106 (2017) 200-204.

[49] B. H. Østerås, E. O. Hole, D. R. Olsen, E. Malinen, EPR dosimetry of radiotherapy photon beams in inhomogeneous media using alanine films, Physics in medicine and biology 51 (24) (2006) 6315.

[50] F. Principato, G. Gerardi, A. Turturici, G. Raso, M. Quartararo, F. Pintacuda, L. Abbene, The" Livio Scarsi" X-Ray Facility at University of Palermo for Device Testing, in: 2015 15th European Conference on Radiation and Its Effects on Components and Systems (RADECS), IEEE, 2015, pp. 1-7.

[51] G. Gerardi, L. Abbene, A digital approach for real time high-rate high-resolution radiation measurements, Nuclear Instruments and Methods in Physics Research Section A: Accelerators, Spectrometers, Detectors and Associated Equipment 768 (2014) 46-54.

[52] L. Abbene, G. Gerardi, High-rate dead-time corrections in a general purpose digital pulse processing system, Journal of synchrotron radiation 22 (5) (2015) 1190-1201.

[53] ASTM E666-14, Standard Practice for Calculating Absorbed Dose From Gamma or X Radiation, Tech. rep., ASTM International, West Conshohocken, PA (2014).

[54] J. M. Boone, A. E. Chavez, Comparison of X-ray cross sections for diagnostic and therapeutic medical physics, Medical physics 23 (12) (1996) 1997-2005.

[55] N. A. Gkanatsios, W. Huda, Computation of energy imparted in diagnostic radiology, Medical physics 24 (4) (1997) 571-579

[56] M. Marrale, M. Brai, G. Gennaro, A. Triolo, A. Bartolotta, M. D’Oca, G. Rosi, Alanine blends for ESR measurements of thermal neutron fluence in a mixed radiation field, Radiation protection dosimetry 126 (1-4) (2007) 631-635.

[57] T. Garcia, M. Lin, I. Pasquié, V. Lourenço, A methodology for choosing parameters for ESR readout of alanine dosimeters for radiotherapy, Radiation Physics and Chemistry 78 (9) (2009) 782-790.

[58] J. Briesmeister, A general Monte Carlo N-Particle Transport Code. Los Alamos National Laboratory Report no.: LA-13709-M., Tech. rep., Los Alamos National Laboratory (2000).

[59] E. Malinen, E. A. Hult, E. O. Hole, E. Sagstuen, Alanine radicals, part 4: Relative amounts of radical species in alanine dosimeters after exposure to 6-19 MeV electrons and $10 \mathrm{kV}-15 \mathrm{MV}$ photons, Radiation 
research 159 (2) (2003) 149-153.

[60] E. Adolfsson, G. A. Carlsson, J.-E. Grindborg, H. Gustafsson, E. Lund, Å. C. Tedgren, Response of lithium formate EPR dosimeters at photon energies relevant to the dosimetry of brachytherapy, Medical physics 37 (9) (2010) 4946-4959.

[61] M. Grotzer, E. Schültke, E. Bräuer-Krisch, J. Laissue, Microbeam radiation therapy: Clinical perspectives, Physica Medica 31 (6) (2015) 564 - 567, radiation Therapy with Synchrotron Radiation: Achievements and Challenges. doi:https://doi.org/10.1016/j.ejmp.2015.02.011.

URL http: //www. sciencedirect.com/science/article/pii/S1120179715000459

[62] D. L. D. Mason, J. J. Battista, R. B. Barnett, A. T. Porter, Ytterbium-169: Calculated physical properties of a new radiation source for brachytherapy, Medical Physics 19 (3) (1992) 695-703. arXiv: https://aapm.onlinelibrary.wiley.com/doi/pdf/10.1118/1.596813, doi:10.1118/1.596813.

URL https : //aapm.onlinelibrary.wiley.com/doi/abs/10.1118/1.596813 